\title{
Erratum: Probing neutron-proton dynamics by pions [Phys. Rev. C 93, 044612 (2016)]
}

\author{
Natsumi Ikeno, Akira Ono, Yasushi Nara, and Akira Ohnishi
}

(Received 1 May 2018; published 19 June 2018)

DOI: 10.1103/PhysRevC.97.069902

The Pauli blocking for the nucleon(s) in the final state of two-particle collisions and particle decays was found to be incorrect with the blocking probability too small by a factor of 4 , in the code of a hadronic cascade model (JAM) employed in our original publication. This affects, in principle, the $N N \leftrightarrow N \Delta$ and $\Delta \rightarrow N \pi$ processes in the main calculations of the original paper which combines the antisymmetrized molecular dynamics (AMD) and JAM. The results of the usual JAM simulations, which are shown mainly as a reference case without a mean field, are also affected.

We revise all the figures (Figs. 1-10) with the corrected numerical results for completeness, although most of the changes are small and do not influence the statements and conclusions in the original paper except for the following point.

The approximate equality $\Delta^{-} / \Delta^{++} \simeq(N / Z)^{2}$ or $(N / Z)_{\rho, p}^{2}$ is not as good as in the original paper because the $\Delta^{-} / \Delta^{++}$ production ratio is now higher than $(N / Z)_{\rho, p}^{2}$. However, the updated results even strengthen our statement since the correlation between $\Delta^{-} / \Delta^{++}$and $(N / Z)_{\rho, p}^{2}$ is stronger than in our paper as seen in Fig. 8 and in the comparison of Figs. 6 and $7($ b). The final $\pi^{-} / \pi^{+}$ratio is also significantly higher than in the original paper.

This problem of Pauli blocking was found during the work for the transport code comparison [1]. The JAM results shown in Ref. [1] were performed after this correction and are therefore not affected.

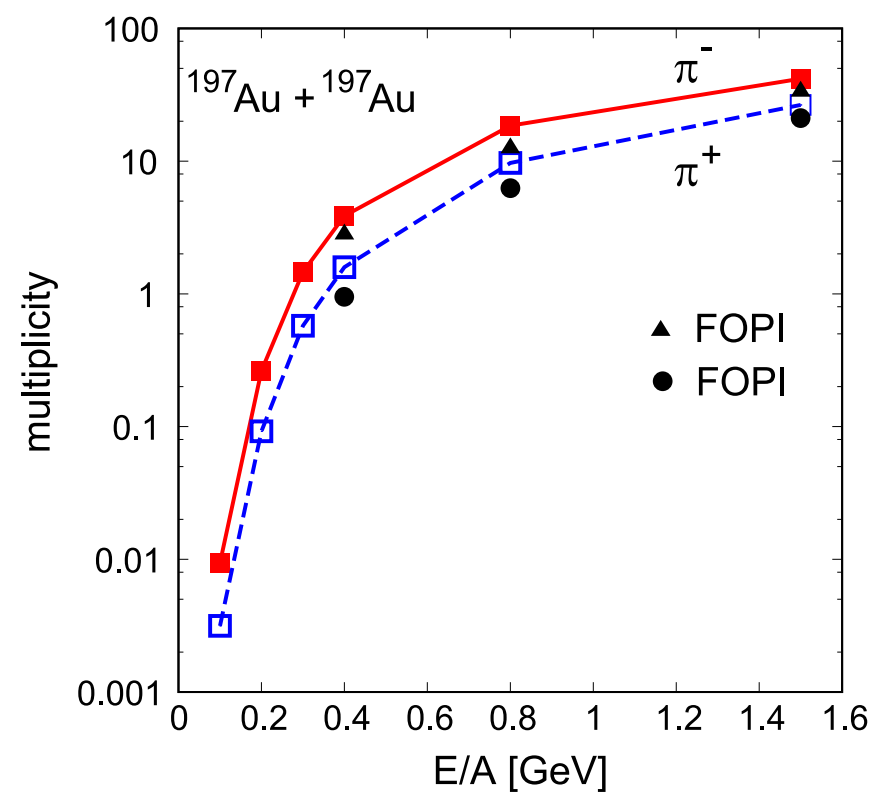

FIG. 1. Revised version of Fig. 1 in the original paper. Incident energy dependence of the charged pion multiplicities in central Au $+\mathrm{Au}$ collisions. The two lines indicate the multiplicities of $\pi^{-}$and $\pi^{+}$, respectively, calculated by JAM. The symbols represent the experimental data of the FOPI Collaboration [2]. 


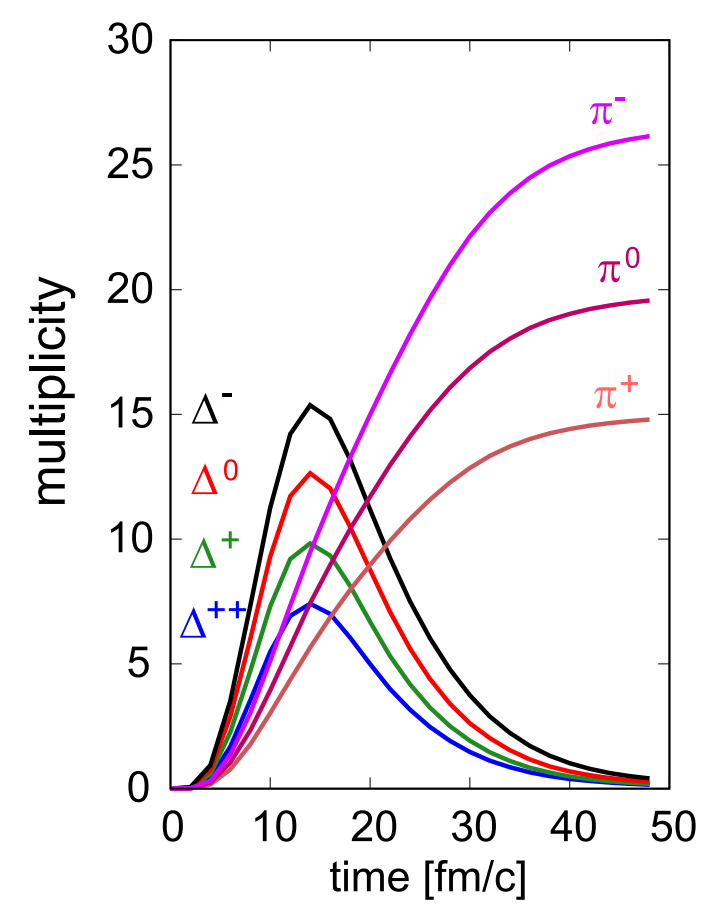

FIG. 2. Revised version of Fig. 2 in the original paper. Time evolution of the numbers of pions and $\Delta$ resonances calculated by JAM for the central $\mathrm{Au}+\mathrm{Au}$ collisions at $1 \mathrm{GeV} /$ nucleon with the impact parameter $b=0$.
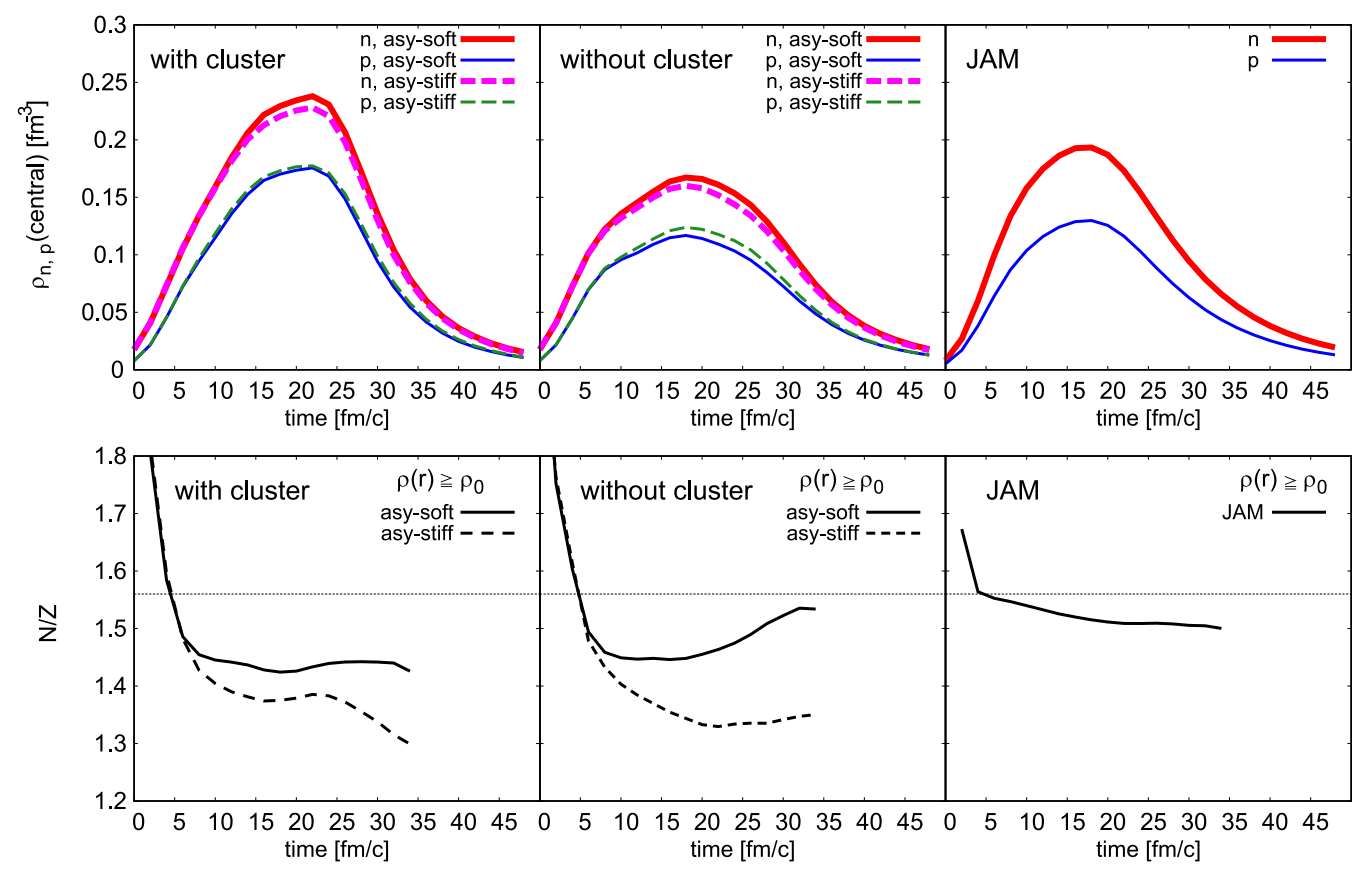

FIG. 3. Revised version of Fig. 3 in the original paper. Time evolution of the neutron and proton densities in the central part of the system within the radius of $2 \mathrm{fm}$ (upper) and the ratio of neutrons and protons (lower) in central collisions of ${ }^{132} \mathrm{Sn}+{ }^{124} \mathrm{Sn}$ at $300 \mathrm{MeV} / \mathrm{nucleon}$. The left and middle panels show the results of AMD+JAM with clusters and without clusters, respectively. The right panels are for the simple JAM calculation. Two different lines (solid and dotted) correspond to the two different density dependences of the symmetry energy. The horizontal lines in the bottom panels represent the ratio of the total system $(N / Z)_{\text {sys }}=1.56$. 


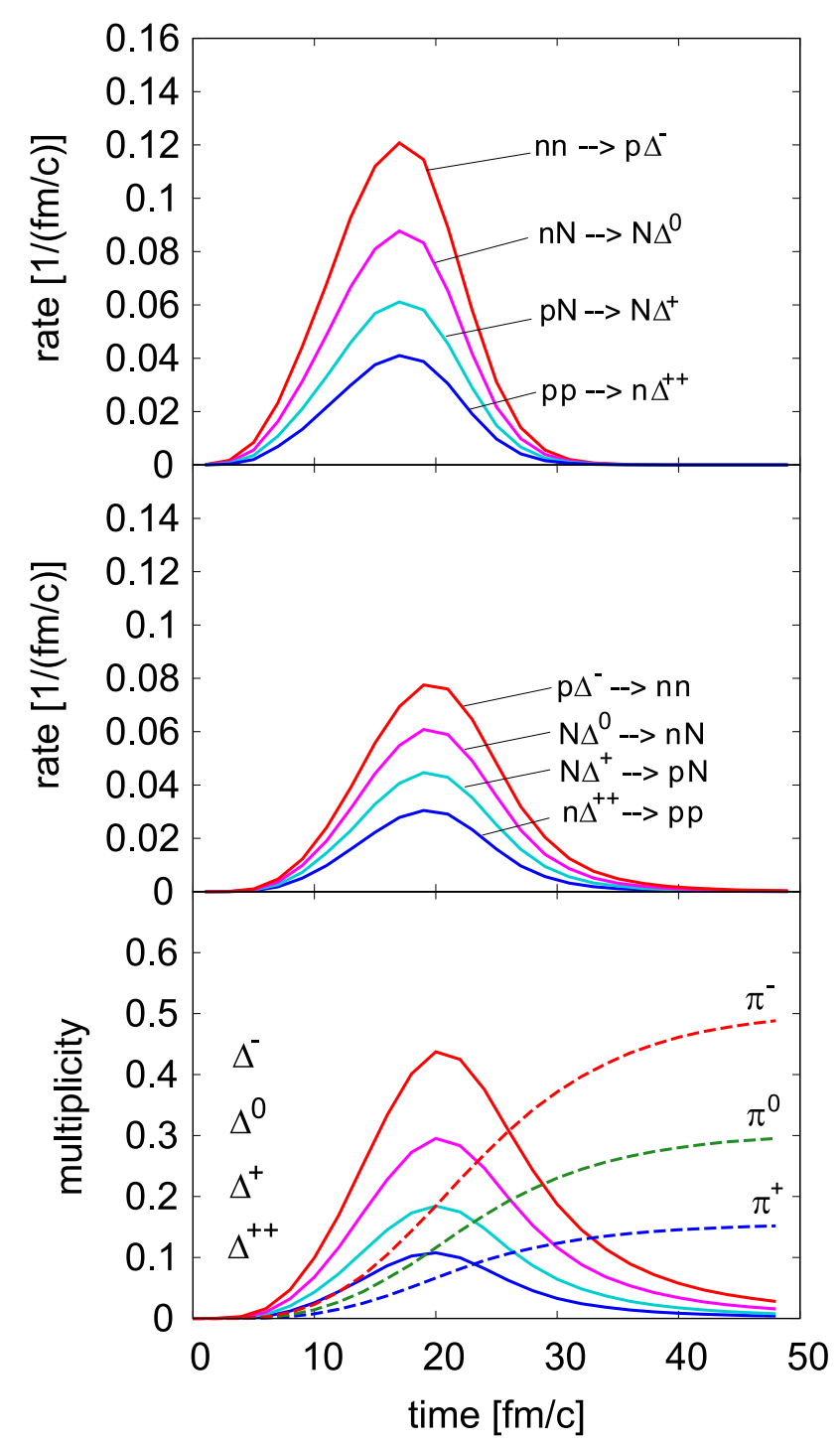

FIG. 4. Revised version of Fig. 4 in the original paper. Reaction rates of $\Delta$ production (upper), $\Delta$ absorption (middle), and the numbers of existing $\Delta$ resonances and pions (lower) as functions of time in the AMD+JAM calculation with clusters (asy-soft) for central collisions of ${ }^{132} \mathrm{Sn}+{ }^{124} \mathrm{Sn}$ at $300 \mathrm{MeV} /$ nucleon. For the production and absorption of $\Delta^{+}$and $\Delta^{0}$, the line shows the sum of the reaction rates with a neutron $(N=n)$ and with a proton $(N=p)$.

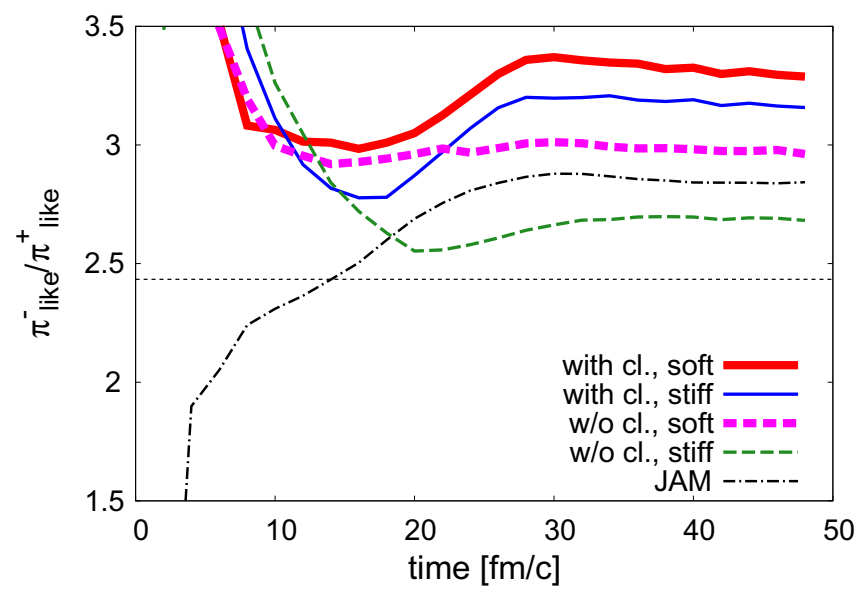

FIG. 5. Revised version of Fig. 5 in the original paper. The time evolution of the $\pi_{\text {like }}^{-} / \pi_{\text {like }}^{+}$ratios in central collisions of ${ }^{132} \mathrm{Sn}+{ }^{124} \mathrm{Sn}$ at $300 \mathrm{MeV} /$ nucleon in the five cases of calculation. The horizontal line represents the $(N / Z)_{\text {sys }}^{2}$ ratio of the total system. 


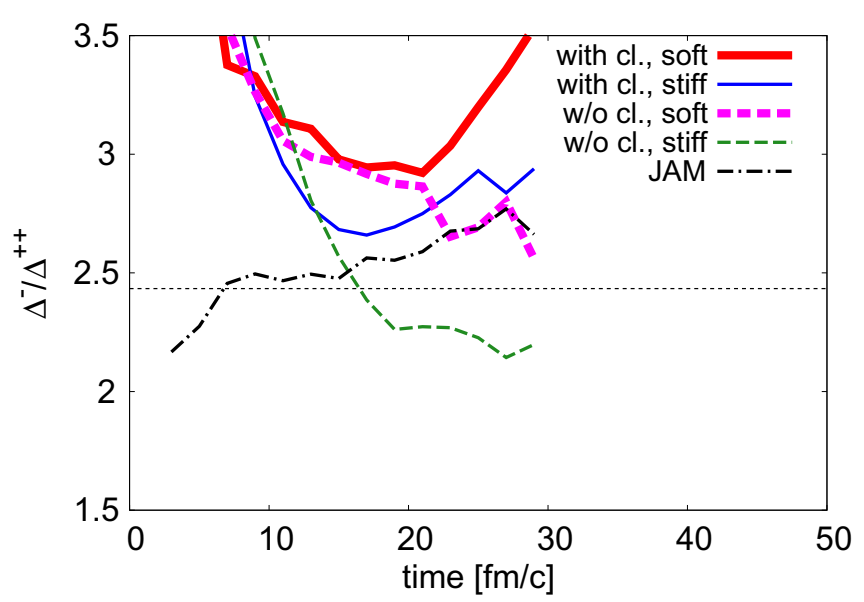

FIG. 6. Revised version of Fig. 6 in the original paper. The time evolution of the $\Delta^{-} / \Delta^{++}$ratio of the $\Delta$ production rates. The five different lines show the calculations. The horizontal line represents the $(N / Z)_{\text {sys }}^{2}$ ratio of the total system.
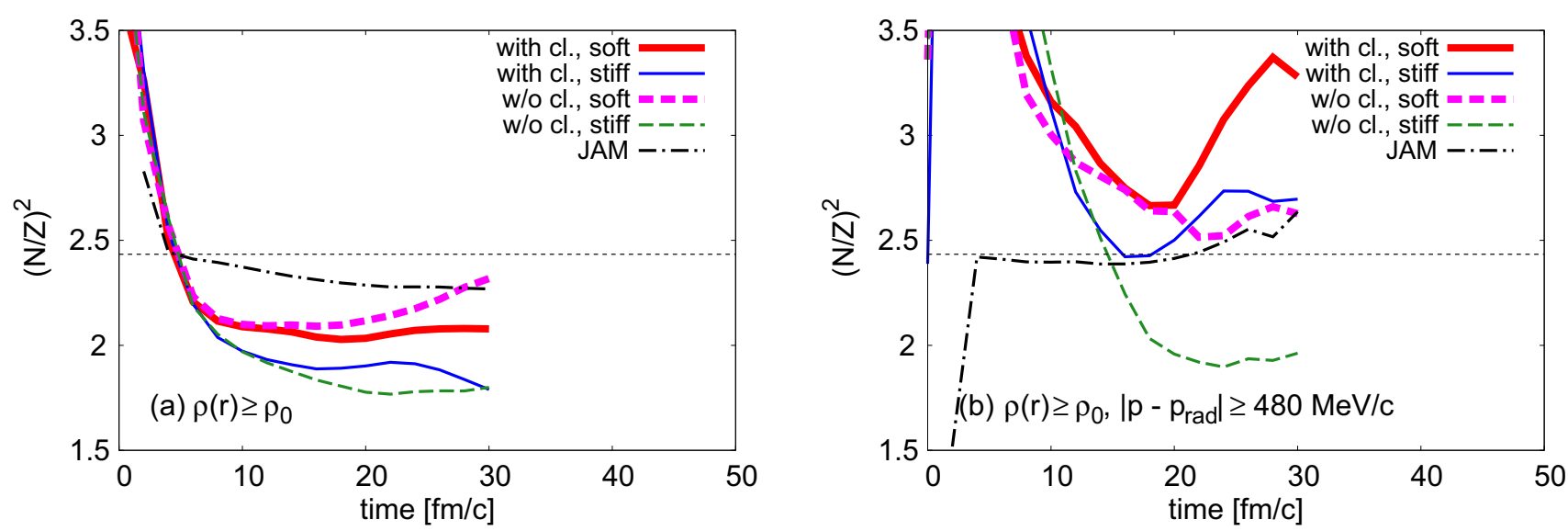

FIG. 7. Revised version of Fig. 7 in the original paper. The time evolution of the squared ratio of neutron and proton $(N / Z)^{2}$. The left and right figures show the $(N / Z)^{2}$ ratio calculated for the nucleons in conditions (a) and (b), respectively. See the details in the text.

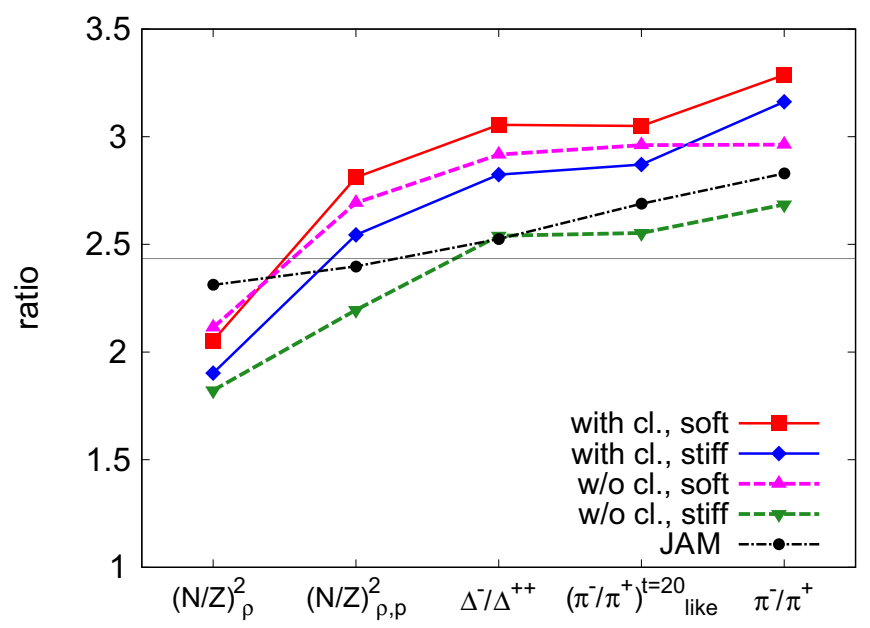

FIG. 8. Revised version of Fig. 8 in the original paper. The nucleon ratios $(N / Z)_{\rho}^{2}$ and $(N / Z)_{\rho, p}^{2}$ [Eq. (25) in our paper] in the high-density region without and with the high-momentum condition, respectively, the $\Delta^{-} / \Delta^{++}$production ratio [Eq. (26) in the paper], the pionlike ratio at $t=20 \mathrm{fm} / c$, and the final $\pi^{-} / \pi^{+}$ratio. Each line connects the ratios for each of the five cases of calculation for central collisions of ${ }^{132} \mathrm{Sn}+{ }^{124} \mathrm{Sn}$ at $300 \mathrm{MeV} /$ nucleon. The horizontal line represents the $\left.(N / Z)\right)_{\text {sys }}^{2}$ ratio of the total system. The statistical uncertainties in the final $\pi^{-} / \pi^{+}$ratio are smaller than 0.03 . 

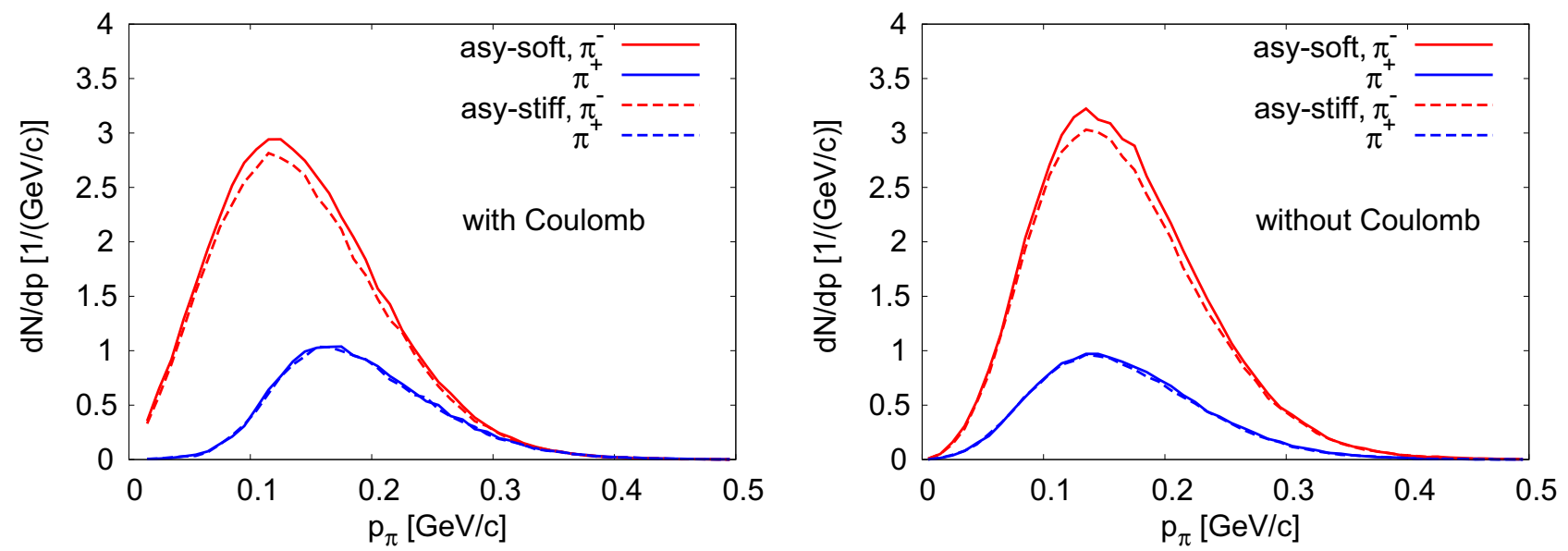

FIG. 9. Revised version of Fig. 9 in the original paper. Momentum distribution of $\pi^{-}$and $\pi^{+}$in the center-of-mass frame calculated with AMD +JAM with clusters for central collisions of ${ }^{132} \mathrm{Sn}+{ }^{124} \mathrm{Sn}$ at $300 \mathrm{MeV} /$ nucleon. The left and right panels show the results with and without Coulomb force for pions, respectively.
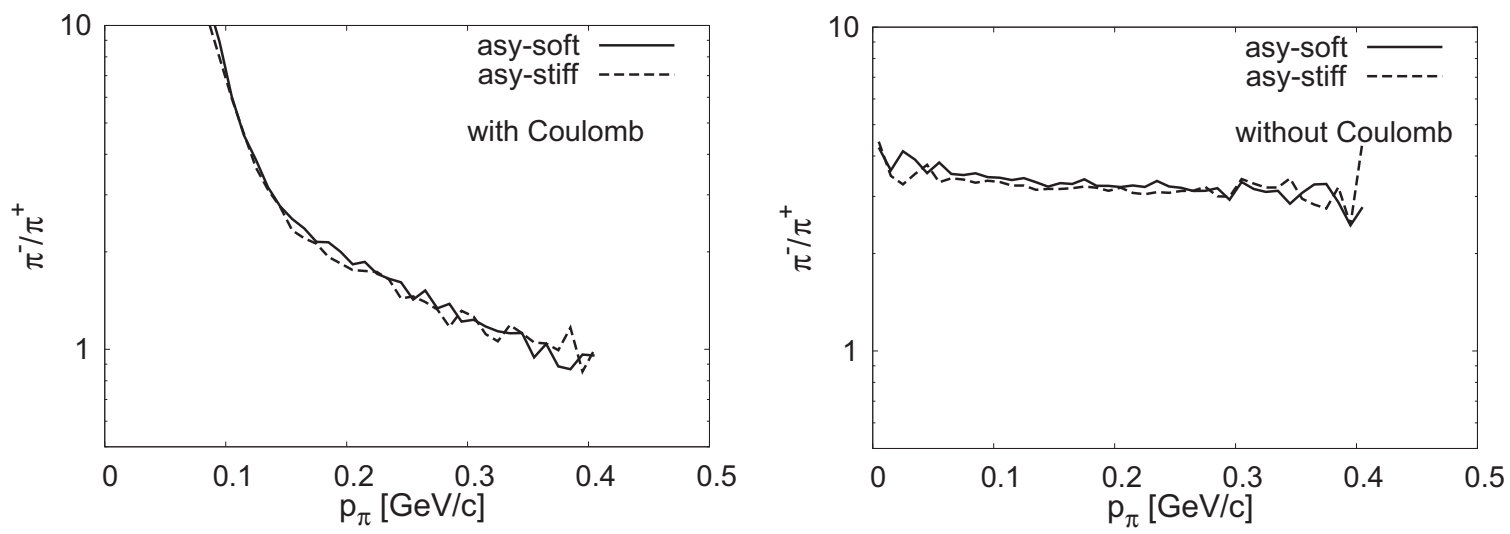

FIG. 10. Revised version of Fig. 10 in the original paper. The same as Fig. 9 but the $\pi^{-} / \pi^{+}$spectral ratio is shown.

[1] Y.-X. Zhang, Y.-J. Wang, M. Colonna, P. Danielewicz, A. Ono, M. B. Tsang, H. Wolter, J. Xu, L.-W. Chen, D. Cozma, Z.-Q. Feng, S. Das Gupta, N. Ikeno, C.-M. Ko, B.-A. Li, Q.-F. Li, Z.-X. Li, S. Mallik, Y. Nara, T. Ogawa, A. Ohnishi, D. Oliinychenko, M. Papa, H. Petersen, J. Su, T. Song, J. Weil, N. Wang, F.-S. Zhang, and Z. Zhang, Phys. Rev. C 97, 034625 (2018).

[2] W. Reisdorf et al. (FOPI Collaboration), Nucl. Phys. A 848, 366 (2010). 